Background Rheumatoid arthritis (RA) is a prototypic autoimmune disease characterised by a prominent humoral autoimmunity. Of particular relevance is the local production of autoantibodies such as rheumatoid factor and anti-citrullinated protein antibodies in the inflamed synovial tissue. The mechanisms underlying break of B cell tolerance and local autoantibody production remains poorly understood.

Objectives To identify cellular and molecular pathways implicated in RA-specific humoral autoimmunity.

Methods Synovial tissue samples were obtained by arthroscopy from untreated individuals with RA $(n=33)$ and inflammation matched SpA controls $(n=58)$. Gene expression profiling was performed on tissue samples of patients with established arthritis using 44K. Whole Genome Human microarrays (Agilent). Top differentially expressed genes were validated on three independent cohorts by Taqman based RT-qPCR and immunohistochemistry. Collagen-induced arthritis (CIA) and Experimental autoimmune encephalomyelitis (EAE) experiments were conducted using Bob1 knockout mice and their littermate controls.

Results Microarray screening for genes differentially expressed in the inflamed synovium, the key target of the disease process in RA, revealed a prominent and disease-specific $B$ cell/plasma cell signature with the B cell-specific transcriptional co-activator Bob1 and its transcriptional target BCMA among the most upregulated genes. Validation by RT-qPCR on two independent cohorts representing early and established arthritis confirmed microarray data and demonstrated elevated expression of Bob1 and BCMA not only in established RA, but also at the early phase of the disease. Quantitative evaluation of immunohistochemical stainings of synovial tissue with monoclonal antibody for Bob1 revealed significant increase in Bob1 positive cells in RA synovium $(p<0.01)$. Next we determined whether lack of functional Bob1 modifies disease onset or severity in CIA. Interestingly, the results showed that Bob1 $1^{-/}$mice were fully resistant to CIA induction compared to their wild-type littermates. This remarkable protection from CIA is explained by decreased antigen-presentation/costimulatory capacity of B cells and by failure to produce pathogenic anti-collagen autoantibodies in the absence of Bob1.

Conclusions The specific increase in Bob1 expressing cells in RA synovitis and the resistance of Bob1-deficient mice to development of CIA indicate that Bob1/BCMA axis may contribute to humoral autoimmunity in RA. The relationship between an aberrant Bob1 expression and the break of peripheral tolerance in RA is currently under investigation.

\section{Microbes and autoimmunity}

\section{A6.1 ANTIBODY RESPONSE AGAINST PORPHYROMONAS GINGIVALIS AND MATRIX METALLOPROTEINASE-3 ARE ASSOCIATED WITH ANTI-CITRULLINATED PROTEIN ANTIBODY IN RHEUMATOID ARTHRITIS, BUT ONLY MATRIX METALLOPROTEINASE-3 IS A PREDICTIVE FACTOR OF RESPONSE TO INFLIXIMAB}

doi:10.1136/annrheumdis-2013-203220.1

${ }^{1} \mathrm{M}$ Rinaudo-Gaujous, ${ }^{2} \mathrm{P}$ Miossec, ${ }^{3} \mathrm{~V}$ Blasco-Baque, ${ }^{4} \mathrm{P}$ Gaudin, ${ }^{5} \mathrm{~T}$ Thomas, ${ }^{1} \mathrm{~A}$ Moreau, ${ }^{1} \mathrm{C}$ Genin, ${ }^{1, *} \mathrm{~S}$ Paul, ${ }^{5, *} \mathrm{H}$ Marotte. 'Laboratory of Immunology and Immunomonitoring, CIC CIE3 Inserm Vaccinology, Hôpital Nord, CHU Saint-Etienne (France); ${ }^{2}$ Clinical Immunology Unit, Departments of Immunology and Rheumatology, Hôpital Edouard Herriot, CHU Lyon (France): ${ }^{3}$ Institute of Cardiovascular and Metabolic Diseases, CHU Rangueil (France); " Grenoble Teaching Hospital, CHU Hôpital Sud, Echirolles (France); ${ }^{5}$ Department of Rheumatology, Hôpital Nord, CHU Saint-Etienne (France),

*The authors contribute equally to this work

Background and Objectives This study evaluates clinical and biological markers of rheumatoid arthritis (RA) severity including matrix metalloproteinase (MMP-3) and Porphyromonas gingivalis (P. gingivalis) serology during infliximab treatment and highlights predictive factors of response to infliximab.
Materials and Methods Joint damage and severe periodontal disease were assessed in 101 RA patients included in this study. DAS28, anti-citrullinated protein antibodies (ACPA), anti-P. gingivalis antibody, and MMP-3 were monitored before and at 6 months of infliximab therapy. ACPA, anti-P. gingivalis antibody, and MMP-3 were determined by ELISA.

Results At baseline, ACPA titers were associated with anti- $P$. gingivalis LPS-specific antibodies titers $(\mathrm{P}<0.05)$. Anti- $P$. gingivalis antibodies were not significantly correlated with clinical, biological, or destruction parameters of RA disease. At 6 months of infliximab therapy, MMP-3 level decreased (from $119 \pm 103 \mathrm{ng} / \mathrm{ml}$ to $62.44 \pm$ $52 \mathrm{ng} / \mathrm{ml} ; \mathrm{P}<0.0001)$, whereas $P$. gingivalis antibody levels remained at the same level. DAS28 and inflammation markers (CRP and ESR) also decreased significantly during infliximab therapy $(\mathrm{P}<0.05)$ as ACPA levels $(\mathrm{P}<0.001)$. Only high MMP-3 level at baseline was associated with infliximab efficacy $(\mathrm{P}<0.01)$.

Conclusions MMP-3 level can be a useful marker of the efficacy of infliximab in RA patients. The treatment did not affect anti$P$. gingivalis antibodies.

\section{A6.2 INDUCIBLE TERTIARY LYMPHOID STRUCTURES AND AUTOIMIMUNITY IN A NOVEL MODEL OF SIALOADENITIS IN WILD-TYPE MICE}

doi:10.1136/annrheumdis-2013-203220.2

'D Lucchesi, 'M Bombardieri, ${ }^{2} \mathrm{~F}$ Barone, ${ }^{2} \mathrm{~S}$ Nayar, ${ }^{3} \mathrm{G}$ Proctor, ${ }^{2} \mathrm{CD}$ Buckley, ${ }^{1} \mathrm{C}$ Pitzalis. ${ }^{1}$ William Harvey Research Institute, Experimental Medicine and Rheumatology, London, UK; 'University of Birmingham, Rheumatology Research Group, Birmingham, UK; ${ }^{3}$ King's College, Oral Medicine and Pathology, London, UK

Introduction Salivary glands of patients with Sjögren's syndrome (SS) develop ectopic lymphoid structures (ELS) characterised by $\mathrm{B} / \mathrm{T}$ cell compartmentalisation, the formation of high endothelial venules (HEV), follicular dendritic cell networks (FDCs), functional $\mathrm{B}$ cell activation with expression of activation-induced cytidine deaminase (AID) as well as local differentiation of autoreactive plasma cells. The mechanisms triggering ELS formation, autoimmunity and exocrine dysfunction in SS are largely unknown. Here we present a novel model of inducible ectopic lymphoid tissue formation, breach of humoral self-tolerance and salivary hypofunction following delivery of a replication-deficient adenovirus-5 (AdV5) in submandibular glands of C57BL/ 6 mice through retrograde excretory duct cannulation.

Materials and Methods Luciferase- or LacZ-encoding Ad5 were delivered in C57BL/6 mice salivary glands (SG) through retrograde cannulation. SGs were collected at various time-points 1, 2 and 3 weeks post-cannulation ( $\mathrm{pc}$ ) and frozen sections were graded for infiltration and stained for T/B cell segregation, FDCs and HEV markers. Submandibular salivary flow was induced by pilocarpine stimulation and the amount of saliva measured. Expression of TLSrelated genes was investigated by TaqMan-PCR. Anti-viral antibodies and autoantibodies were detected by IF and western blot.

Results In this model, inflammation rapidly and consistently evolves from diffuse infiltration towards the development of SS-like periductal lymphoid aggregates within 2 weeks from AdV delivery. These infiltrates progressively acquire ELS features and support functional GL7+/AID + germinal centres. Formation of ELS is preceded by ectopic expression of lymphoid chemokines CXCL13, CCL21 and CCL19 as well as lymphotoxin- $\beta$ and is associated with development of anti-nuclear antibodies in $75 \%$ of the mice. Finally, reduction in salivary flow was observed over 3 weeks post AdV infection consistent with exocrine gland dysfunction as a consequence of the inflammatory response.

Conclusions This novel model has the potential to unravel the cellular and molecular mechanisms regulating ELS formation and their role in exocrine dysfunction and autoimmunity in SS. 